\title{
Influence de la fertilisation à base des déchets humains recyclés, des engrais minéraux et de leur combinaison sur le comportement de 3 variétés de maïs (Zea mays L.)
}

Luciens Nyembo Kimuni, llunga Tabu Hugues, Emmanuel Muyambo Musaya, Augustin Ekondo Okese, Mick Assani Bin Lukangila, Antoine Kanyenga Lubobo, Michel Mpundu Mubemba, Louis Baboy Longanza

Faculté des Sciences Agronomiques, Université de Lubumbashi, Lubumbashi, BP 1825 RD Congo

Correspondance : luciensnyembokimuni@gmail.com; michelmpundu@gmail.com

Original submitted in on $11^{\text {th }}$ February 2014. Published online at www.m.elewa.org on $31^{\text {st }}$ May 2014. http://dx.doi.org/10.4314/jab.v77i1.2

\section{RÉSUMÉ}

Objectifs : L'utilisation des déchets urbains solides en agriculture urbaine est une pratique courante. Dans le cas des cultures céréalières, les déchets sont apportés avec peu ou sans engrais minéral et leurs effets sur les cultures et sur le sol sont cependant peu connus. Ce travail a été initié en vue d'évaluer les effets des apports combinés de déchets humains recyclés et des engrais minéraux sur le rendement du maïs. Méthodologie et résultats : L'essai a été installé suivant un dispositif factoriel à trois répétitions. Les effets de quatre doses de déchets humains recyclés $\left(0 ; 1,75 ; 3,5\right.$ et 7 tha-1 $\left.{ }^{-1}\right)$ ont été évalués sur trois variétés de maïs (Babungo, Unilu et Katanga). Les déchets étaient apportées seuls ou avec un supplément d'engrais

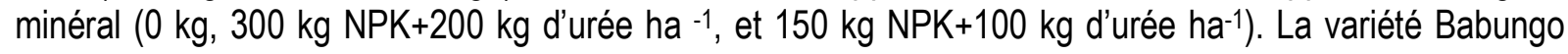
adonné le rendement le plus élevé $\left(6,6\right.$ tha $\left.^{-1}\right)$ que les deux autres variétés qui ont donné des rendements similaires statistiquement $\left(5,3\right.$ t.ha- $^{-1}$ pour la variété Katanga et 4,4 t.ha-1 $^{-1}$ pour Unilu). Comparés au rendement obtenu sur les parcelles fertilisées aux déchets humains ou aux engrais minéraux seuls, les rendements ont été accrus sur les parcelles où les deux fertilisants ont été combinés, hormis la faible dose des déchets humains recyclés combinés aux engrais minéraux (quelle que soit la dose) ou la dose intermédiaire des déchets humains recyclés fèces humaines combinées à la dose de référence des engrais minéraux réduite de moitié.

Conclusion et application: La rentabilité des doses élevées des déchets humains recyclés appliqués seuls ou combinés aux engrais minéraux est comparable à celle obtenue avec les engrais minéraux appliqués seuls (traitements rentables). En revanche, la faible dose de déchets humains recyclés appliquées seule qui a donné une rentabilité excellente (Rapport valeur sur Cout=7,73). La population Katangaise étant en pleine expansion, ceci entraine une augmentation des besoins alimentaires parallèlement à une production élevée des déchets et effluents. Le recyclage de déchets participe à l'assainissement environnemental et la lutte contre la pauvreté par l'accroissement des rendements.

MOTS CLES : Maïs, déchets humains recyclés, rendement, engrais minéraux 
ABSTRACT

Influence of fertilization based on recycled human waste, mineral fertilizers and their combination on the behavior of three varieties of maize (Zea mays L.)

Objectives: The use of municipal solid waste in urban agriculture is a common practice. Recycled human waste made with little or no mineral fertilizers and their effects on crops and soil has however been poorly understood. This work was initiated to evaluate the effects of the combined inputs of recycled human waste and mineral fertilizers on the yield of maize. Methodology and Results: The trial was installed following a factorial design with three replications. The effects of four doses of recycled human waste $(0,1.75,3.5$ and

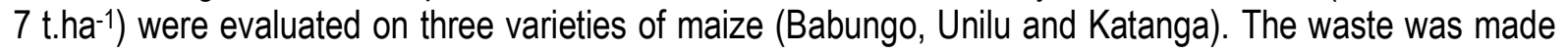
alone or with a mineral fertilizer supplements $\left(0 \mathrm{~kg}, 300 \mathrm{kgNPK}+200 \mathrm{~kg}^{\mathrm{N} e a} \mathrm{ha}^{-1}, 150 \mathrm{~kg} \mathrm{NPK}+100 \mathrm{~kg}\right.$ urea ha-1). The Babungo variety gave the highest yield $\left(6.6\right.$ tha $\left.^{-1}\right)$ compared to the other two varieties who gave similar yields statistically ( 5.3 tha- $^{-1}$ for Katanga variety and 4.4 tha- ${ }^{-1}$ for Unilu). Compared to the yield obtained on plots fertilized with human waste or mineral fertilizers alone, yields were increased on plots where the two fertilizers were combined.Conclusion and Application: The profitability of high doses of recycled human waste applied alone or combined with mineral fertilizers is comparable to that obtained with mineral fertilizers applied alone (profitable treatments). In contrast, the low dose of recycled human wastes applied only to give excellent profitability (Ration Value by Cost= 7.73). Katangese has a growing population, this leads to an increase in food needs along with high production of waste and effluents. Recycling of waste involved in environmental sanitation and the fight against poverty through increased yields.

KEYWORDS: maize, recycled human waste, yield, mineral fertilizers

\section{INTRODUCTION}

Avec le riz (Oryza sativa L.) et le blé tendre (Triticum aestivum L.), le maïs (Zea mays L.) est l'une des trois céréales les plus cultivées au monde. II est cultivé pour l'alimentation humaine et animale mais aussi pour de nombreuses utilisations dans l'industrie textile, pharmaceutique, dans la production de plastique biodégradable et de biocarburant. En ce qui concerne la consommation humaine au Mexique ou en Afrique du sud par exemple, les chiffres peuvent atteindre 50 à + de $10 \mathrm{~kg} / \mathrm{an} /$ par personne de maïs consommé, d'où l'importance du maïs dans la production mondiale (Anzala, 2006). Au Katanga particulièrement à Lubumbashi, la grande production est consommée sous forme de farine et constitue ainsi la nourriture de base préférée par la majorité de la population. Dans le cas de la Province du Katanga, la demande globale en 2008, était de 988.156 tonnes, et l'offre de 500.854 tonnes, soit un rapport offre/demande de plus au moins 0,5 (Nyembo, 2010). Au Katanga, la culture de maïs est pratiquée sur des grandes superficies par des paysans (Nyembo, 2010). Ce mode d'exploitation, combiné avec la croissance démographique (4,1\% par an ; GROUPE HUIT, 2009) et les perturbations climatiques de ces dernières années, a pour conséquence une pression foncière de plus en plus marquée. Cela a entraîné la réduction de la durée de la jachère, qui passe de 10-15 ans à 3 ans voire 2 ans (Kasongo, 2008). Ces contraintes ont contribué à la baisse de la fertilité des sols et à celle du rendement. Pour pallier à cette baisse de fertilité, il faudra recommander entre autre, la fertilisation minérale par les engrais chimiques, la fertilisation organique, de l'utilisation des techniques culturales par la pratique des associations culturales et des rotations et des assolements. Ces différentes propositions ont une incidence financière que ne peut supporter le paysan qui pratique une agriculture de subsistance (Gala et al., 2011; Kasongo et al., 2013). Les agriculteurs en zone urbaine ou périurbaine sont cependant confrontés à la disponibilité de ressources organiques pour développer et augmenter la production agricole. Parallèlement, les villes offrent des ressources organiques variées (N'Dienor, 2006). Les excréta humains sont très riches en nutriments et 


\section{Kimuni et al. J. Appl. Biosci. 2014. Influence de la fertilisation a base des déchets humains recycles,}

des engrais minéraux et de leur combinaison sur le comportement de maïs

permettent d'obtenir des rendements compétitifs à ceux obtenus avec la fumure minérale en culture maraîchère et céréalière (Kiba, 2005). Les études conduites en milieu naturel et en serre (laboratoire) ont montré que les ressources locales comme les déchets organiques et les roches naturelles, appliquées aux sols tropicaux pauvres et acides peuvent fournir les éléments nutritifs nécessaires pour l'alimentation et la croissance des plantes et par conséquent, accroître le rendement des plantes cultivées (Mulaji, 2011). Les déchets humains recyclés constituent un bon substitut au fumier et surtout aux engrais, et il produit des

\section{MATERIELS ET METHODES}

Milieu: L'étude a été menée à la ferme Kassapa, station de recherche de l'Université de Lubumbashi (altitude de $1244 \mathrm{~m}$, latitude $11^{\circ} 37^{\prime} \mathrm{S}$ et une longitude de $27^{\circ} 27^{\prime}$ E) au cours de la saison agricole 2010-2011. Le climat est du type tropical sec avec alternance d'une saison de pluies et d'une saison sèche et les pluviométries enregistrées sont de de $1270 \mathrm{~mm}$ en moyenne (avec une saison de pluie de 118 jours) alors que la température moyenne anuelle est d'environ $20^{\circ} \mathrm{C}$ avec une grande stabilité interannuelle. Le taux d'humidité moyenne est de $62 \%$ avec un niveau d'humidité minimum moyenne de $52 \%$ en saison sèche (juin - août) et un maximum de $80 \%$ durant la saison pluvieuse (novembre - mai). Les sols de Lubumbashi et ses environs sont dominés, par les sols ferralitiques jaune, ocre-jaune et rouge suivant la position topographique et le drainage (Nyembo, 2010). Les analyses du sol ont donné les résultats suivants: $3,27 \% \mathrm{~N}, 0,48 \mathrm{P} 2 \mathrm{O} 5$ et $5,13 \% \mathrm{~K} 2 \mathrm{O}$.

Matériel : Le matériel végétal utilisé est composé de trois variétés de maïs (Babungo, unilu et Katanga) vulgarisée et cultivée dans la région de Lubumbashi. Les déchets humains recyclés utilisés comme fertilisant organique, provenaient des fosses septiques des cités universitaires et ont été hygiénisés (Useni et al., 2013). Elles ont été compostées pendant 14 mois et leur composition, déterminée à partir de la matière sèche, indique $6,5 \%$ de $\mathrm{N}, 0,12 \%$ de P2O5, 2,53 \% de $\mathrm{K} 2 \mathrm{O}$. L'engrais minéral renfermant les éléments $\mathrm{N}, \mathrm{P}$, et $\mathrm{K}$ de formule 10-20-10 et l'urée ( $46 \% N$ ) ont été utilisés.

Dispositif expérimental, traitements et conduite de l'essai: L'essai a été installé suivant un dispositif factoriel. Les traitements, en trois répétitions, consistaient en trois variétés de maïs (Babungo, Unilu et Katanga) et trois doses de fèces humaines $(1,75$; rendements élevés que ceux des pratiques paysannes (Useni et al., 2013) et son intérêt s'accroît avec l'augmentation du prix des engrais. Useni et al. (2013) évaluant les effets de 5 doses des déchets humains recyclés (témoin ou contrôle, 1 t.ha-1, 10 tha $^{-1}, 20$ t.ha $^{-1}, 30$ t.ha-1 $^{-1}$ et 50 t.ha $^{-1}$ ), ont montré que seules les doses élevées de déchets (30 et 50 t.ha-1)ont permis d'accroitre significativement le rendement. L'objectif de ce travail est celui d'évaluer les effets combinés de faibles doses de déchets et des engrais minéraux en vue d'optimiser le rendement du maïs.

3,5 et 7 t.ha $\left.^{-1}\right)$. Les fèces humaines étaient apportées seuls ou avec un supplément d'engrais minéral composé et azoté correspondant à $300 \mathrm{~kg} \mathrm{NPK}+200 \mathrm{~kg}$ d'urée ha ${ }^{-1}$ et $150 \mathrm{~kg} \mathrm{NPK}+100 \mathrm{~kg}$ d'urée ha $^{-1}$. Ces traitements issus des combinaisons des doses des fèces humaines et des engrais chimiques étaient comparés aux témoins sans engrais minéral et avec engrais minéral. Les travaux du sol (labour et hersage) ont été effectués à l'aide des machines agricoles à une profondeur d'environ $30 \mathrm{~cm}$. Le semis est intervenu en date du 20/12/ 2010, aux écartements de 0,75 m x 0,25 $\mathrm{m}$, soit une densité de 53333 plants/ha. II est à noter que l'épandage des engrais minéraux est intervenu 15 jours après semis pour le NPK, à une dose de 300 $\mathrm{kg} / \mathrm{ha}$ ou $150 \mathrm{~kg} \mathrm{ha}^{-1}$ et à 30 jours après semis pour l'urée soit, $200 \mathrm{~kg} \mathrm{ha}^{-1}$ ou $100 \mathrm{~kg} \mathrm{ha}^{-1}$ suivant les traitements. Au cours de l'essai, le taux de levée, les jours à la floraison mâle et la hauteur de plantes à la floraison mâle ont été enregistrés. A la récolte, le nombre d'épis par plante, le nombre de rangées de grains par épi, le poids des grains par épi, le poids de 1000 grains, le rendement en maïs grain et la rentabilité économique ont été déterminés.

Analyse statistique et économique des données: Les données brutes sur les paramètres végétatifs et de rendement ont été traitées par l'analyse de la variance (ANOVA) avec test post hoc (test de DUNCAN) pour la séparation des moyennes. Pour l'analyse économique des traitements, le rapport valeur sur coût (RVC) à été utilisé et pour le calcul du bénéfice, les charges suivantes ont été retenues: l'achat des engrais minéraux, la collecte des fumiers de poules, le transport et l'incorporation dans le sol. Le coût des engrais chimiques est celui observé sur le marché local ( $55 \$ / 50 \mathrm{~kg}$ pour le NPK et $50 \$ / 50 \mathrm{~kg}$ pour l'urée). Le 


\section{Kimuni et al. J. Appl. Biosci. 2014. Influence de la fertilisation a base des déchets humains recycles,}

des engrais minéraux et de leur combinaison sur le comportement de maïs

coût de la main-d'œuvre pour collecter, transporter et incorporer les fumiers de poules ont été évalués à $48 \$$ alors que le transport et l'épandage des engrais minéraux ont été évalué à $10 \$ / 50 \mathrm{~kg}$. Le prix moyen d'une tonne de maïs dans les différents marchés de Lubumbashi est d'environ $250 \$$ (Useni et al., 2013). Le RVC compare la rentabilité des nouveaux traitements au traitement de référence bien connu par les paysans.

\section{RESULTATS}

Les trois variétés de maïs expérimentées ont montré de comportements différents pour tous les paramètres observés (tableau 1). Les variétés Katanga et Unilu ont donné des taux de levée et de résistance à la verse supérieure et similaire que la variété Babungo qui
C'est donc le rapport entre l'augmentation du rendement du nouveau traitement et le coût de fertilisant du même traitement: RVC= valeur de l'augmentation du rendement/coût du fertilisant. Si le rapport valeur/coût dépasse 1 , l'engrais est retable, mais la rentabilité est excellente lorsque RVC est $\geq$ à 3 (FAO, 2000).

donne cependant des plants de maïs de grande taille. La variété Babungo a donné le rendement le plus élevé que les deux autres variétés qui ont donné des rendements similaires statistiquement.

Tableau 1 : Influence de la variété sur le comportement du maïs.

\begin{tabular}{llllll}
\hline Paramètres & \multicolumn{3}{c}{ Variétés } & $\boldsymbol{P}$ & CV \\
& Babungo & Katanga & Unilu & & \\
\hline Taux de levée (\%) & $74,7 \pm 17,4 \mathrm{~b}$ & $86 \pm 9,1 \mathrm{a}$ & $88,2 \pm 9,1 \mathrm{a}$ & 0,001 & 14,9 \\
Hauteur des plantes à l'inflorescence mâle $(\mathrm{cm})$ & $208,2 \pm 41,9 \mathrm{a}$ & $139,9 \pm 39,8 \mathrm{c}$ & $174,9 \pm 16,6 \mathrm{~b}$ & 0,000 & 19,9 \\
Jours à la floraison mâle & $70 \pm 3,3 \mathrm{a}$ & $71,2 \pm 4,8 \mathrm{a}$ & $65,9 \pm 4 \mathrm{~b}$ & 0,001 & 6 \\
Résistance à la verse (\%) & $85,5 \pm 7,9 \mathrm{c}$ & $89,8 \pm 13,3 \mathrm{a}$ & $92,3 \pm 4 \mathrm{a}$ & 0,001 & 10,9 \\
Poids de grains par épi $(\mathrm{g})$ & $183,5 \pm 41,6 \mathrm{a}$ & $159,1 \pm 37,5 \mathrm{~b}$ & $134,2 \pm 22,4 \mathrm{c}$ & 0,001 & 21,7 \\
Poids de 1000 grains $(\mathrm{g})$ & $417,3 \pm 47,6 \mathrm{a}$ & $344 \pm 59,6 \mathrm{~b}$ & $363,3 \pm 47,9 \mathrm{a}$ & 0,001 & 13,9 \\
Rendement (t.ha-1) & $6,6 \pm 2,4 \mathrm{a}$ & $5,3 \pm 2,1 \mathrm{~b}$ & $4,4 \pm 1,6 \mathrm{~b}$ & 0,004 & 38,4 \\
\hline
\end{tabular}

Moyennes \pm écart-type. Les différentes lettres indiquent de différences significatives après le test de DUNCAN. CV : coefficient de variation ; $P$ : Probabilité

L'apport des doses croissantes des déchets humains recyclés seuls ou combinés aux engrais minéraux n'a pas influencé la croissance des variétés de maïs étant donné qu'aucune différence significative n'a été décelée entre les traitements pour tous les paramètres végétatifs observés (tableau 2).

Tableau 2 : Effets des doses croissantes des déchets humains recyclés, engrais minéraux et de leur combinaison sur la croissance du maïs.

\begin{tabular}{|c|c|c|c|c|}
\hline $\begin{array}{l}\text { Types et doses de } \\
\text { fertilisants }\end{array}$ & $\begin{array}{l}\text { Taux de } \\
\text { levée (\%) }\end{array}$ & $\begin{array}{c}\text { Hauteur de plantes à } \\
\text { l'inflorescence mâle }(\mathrm{cm})\end{array}$ & $\begin{array}{c}\text { Jours à la } \\
\text { floraison mâle }\end{array}$ & $\begin{array}{c}\text { Résistance à la } \\
\text { verse (\%) }\end{array}$ \\
\hline Témoin & $85,9 \pm 7,4 a$ & $141,5 \pm 27,6 a$ & $69,2 \pm 3,5 a$ & $78,6 \pm 12,3 a$ \\
\hline 150kg NPK+100 kg urée & $83,6 \pm 5,9 a$ & $153,5 \pm 18 a$ & $68,3 \pm 4,6$ & $84,8 \pm 10,9 a$ \\
\hline $300 \mathrm{~kg}$ NPK+200kg Urée & $80,7 \pm 17,9 a$ & $178,1 \pm 46 a$ & $69,2 \pm 4,7 a$ & $86,9 \pm 7,1 \mathrm{a}$ \\
\hline 3,5 tha-1 DHR & $76,4 \pm 17,5 a$ & $156,5 \pm 21,5 a$ & $69,3 \pm 5,5 a$ & $80,5 \pm 19 a$ \\
\hline 7 t.ha-1 $^{-1} \mathrm{DHR}$ & $89,2 \pm 23,7 a$ & $162,8 \pm 18,6 a$ & $69,3 \pm 2,4 a$ & $84,8 \pm 15,5 a$ \\
\hline 1,75 tha-1 DHR & $90,8 \pm 5,2 a$ & $176,5 \pm 36 a$ & $69,8 \pm 4,8 a$ & $82 \pm 15,5 a$ \\
\hline $\begin{array}{l}3,5 \text { t.ha-1 }^{-1} \mathrm{DHR}+300 \mathrm{~kg} \\
\text { NPK+200kg Urée }\end{array}$ & $86 \pm 8,4 a$ & $180,6 \pm 51 a$ & $66,4 \pm 5,2 a$ & $85 \pm 9,9 a$ \\
\hline 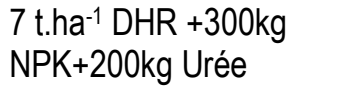 & $81,7 \pm 13,9 a$ & $199,2 \pm 33,4 a$ & $69,7 \pm 4,6 a$ & $86 \pm 12,3 a$ \\
\hline $\begin{array}{l}1,75 \text { t.ha-1 }^{-1} \mathrm{DHR}+300 \mathrm{~kg} \\
\text { NPK+200kg Urée }\end{array}$ & $79,8 \pm 11,9 a$ & $173,9 \pm 62,9 a$ & $64,3 \pm 5,7 a$ & $86,3 \pm 11,8 a$ \\
\hline 3,5 tha-1 DHR +150kg & $76,8 \pm 16,4 a$ & $174,8 \pm 66,2 a$ & $67 \pm 4,7 a$ & $87,8 \pm 11,9 a$ \\
\hline
\end{tabular}


Kimuni et al. J. Appl. Biosci. 2014. Influence de la fertilisation a base des déchets humains recycles, des engrais minéraux et de leur combinaison sur le comportement de maïs

\begin{tabular}{l|c|c|c|c}
\hline NPK+100kg Urée & $84,7 \pm 10,7 a$ & $191,5 \pm 50,5 a$ & $66,8 \pm 4,2 a$ & $88,5 \pm 12,7 a$ \\
7 t.ha-1 DHR+150kg & & & & \\
NPK+100kg Urée & $87,9 \pm 5,9 a$ & $189,6 \pm 38,9 a$ & $68,6 \pm 2,7 a$ & $86,9 \pm 10,4 a$ \\
1,75 t t.ha- ${ }^{-1}$ DHR +150kg & & & & \\
NPK+100kg Urée & 16,6 & 25,1 & 0,6 & 15,4 \\
\hline CV & 0,431 & 0,271 & 0,126 & 0,86 \\
$P$ &
\end{tabular}

Moyennes \pm écart-type. Les différentes lettres indiquent de différences significatives après le test de DUNCAN. DHR : Déchets humains recyclés; $C V$ : coefficient de variation ; $P$ : Probabilité

Les déchets humains recyclés appliqués seuls ou combinés aux engrais minéraux ont influencé les paramètres de rendement, hormis le poids de 1000 graines. La dose de 7 tonnes de déchets humains recyclés combinés à la dose de référence des engrais minéraux réduite de moitié ou appliquée comme telle a accru significativement le poids de grains par épi, tout comme la dose de référence des engrais minéraux combinée à la dose intermédiaire des déchets humains recyclés (tableau 3). En outre, les parcelles fertilisées à la dose de 7 tonnes des déchets humains recyclés combinés à la dose de référence des engrais minéraux a produit des épis à poids de grains élevé, suivies des parcelles fertilisées aux engrais minéraux seuls. Comparés au rendement obtenu sur les parcelles fertilisées aux déchets humains ou aux engrais minéraux seuls, les rendements ont été accrus sur les parcelles où les deux fertilisants ont été combinés, hormis la faible dose des déchets humains recyclés combinés aux engrais minéraux (quelle que soit la dose) ou la dose intermédiaire des déchets humains recyclés fèces humaines combinées à la dose de référence des engrais minéraux réduite de moitié.

Tableau 3 : Effets des doses croissantes des déchets humains recyclés, engrais minéraux et de leur combinaison sur les paramètres de rendement du maïs.

\begin{tabular}{|c|c|c|c|}
\hline Types et doses de fertilisants & $\begin{array}{l}\text { Poids de grains par } \\
\text { épi }\end{array}$ & $\begin{array}{l}\text { Poids de } 1000 \\
\text { grains }\end{array}$ & $\begin{array}{l}\text { Rendement en } \\
\text { maïs grain }\end{array}$ \\
\hline Témoin & $110,6 \pm 26 \mathrm{e}$ & $340,1 \pm 40 a$ & $2,1 \pm 1,2 f$ \\
\hline 150kg NPK+100 kg Urée & $114,7 \pm 14,9 e$ & $354,4 \pm 21,7 a$ & $3,7 \pm 0,8 \mathrm{e}$ \\
\hline $300 \mathrm{~kg}$ NPK+200kg Urée & $173,9 \pm 27,7 b$ & $397,8 \pm 67,9 a$ & $5,9 \pm 1,3 c$ \\
\hline $3,5 \mathrm{tEH}$ & $124,4 \pm 29,7 d$ & $336 \pm 60,4 a$ & $3,9 \pm 1,3 e$ \\
\hline $7 \mathrm{tEH}$ & $144,4 \pm 14,3 c$ & $375,6 \pm 55 a$ & $4,5 \pm 1,4 \mathrm{e}$ \\
\hline $1,75 \mathrm{t} \mathrm{EH}$ & $140,5 \pm 28,7 \mathrm{~cd}$ & $352,2 \pm 69 a$ & $4,7 \pm 1,7 e$ \\
\hline $3,5 \mathrm{t}$ EH +300kg NPK+200kg Urée & $177,7 \pm 33,8 \mathrm{ab}$ & $394,4 \pm 58,7 a$ & $6,8 \pm 1,8 b$ \\
\hline $7 \mathrm{t} \mathrm{EH}+300 \mathrm{~kg} N P K+200 \mathrm{~kg}$ Urée & $192,9 \pm 40,1 \mathrm{a}$ & $400 \pm 52,9 a$ & $7,7 \pm 2,4 a$ \\
\hline 1,75 t EH +300kg NPK+200kg Urée & $169,7 \pm 19,9 b c$ & $362,2 \pm 44,9 a$ & $5,8 \pm 1,3 c$ \\
\hline $3,5 \mathrm{t}$ EH +150kg NPK+100kg Urée & $166,1 \pm 41,3 b c$ & $405,6 \pm 54,7 a$ & $5,6 \pm 1,5 \mathrm{~cd}$ \\
\hline $7 \mathrm{t} \mathrm{FH}+150 \mathrm{~kg}$ NPK+100kg Urée & $182,8 \pm 41,1 a b$ & $394,4 \pm 71,7 a$ & $7,3 \pm 1,9 a b$ \\
\hline 1,75 t FH +150kg NPK+100kg Urée & $165 \pm 44,7 b c$ & $364,4 \pm 60,2 a$ & $5,3 \pm 2,3 d$ \\
\hline CV & 20,7 & 15,6 & 32 \\
\hline$P$ & 0,0001 & 0,103 & 0,0000 \\
\hline
\end{tabular}

Moyennes \pm écart-type. Les différentes lettres indiquent de différences significatives après le test de DUNCAN. DHR : Déchets humains recyclés ; CV : coefficient de variation ; $P$ : Probabilité

Les résultats du tableau 4 montrent que la rentabilité des doses élevées des déchets humains recyclés appliqués seuls ou combinés aux engrais minéraux est comparable à celle obtenue avec les engrais minéraux appliqués seuls (traitements rentables). En revanche, la faible dose de fèces humaines appliquées seule qui a donné une rentabilité excellente (RVC=7,73). 
Tableau 4 : Analyse économique de différents traitements. DHR : Déchets humains recyclés

\begin{tabular}{llllllll}
\hline Traitements & $\begin{array}{l}\text { Coût } \\
\text { engrais } \\
\text { chimique } \\
\text { (\$/ha) }\end{array}$ & $\begin{array}{l}\text { Coût des } \\
\text { DHR } \mathbf{( \$ / h a )})\end{array}$ & $\begin{array}{l}\text { Coût total } \\
\text { de } \\
\text { fertilisants } \\
\text { (\$/ha) }\end{array}$ & $\begin{array}{l}\text { Rendement } \\
\text { en maïs } \\
\text { grain } \\
\text { (t.ha-1) }^{-1}\end{array}$ & $\begin{array}{l}\text { Augmentation } \\
\text { du rendement } \\
\text { (t.ha-1) }\end{array}$ & $\begin{array}{l}\text { Valeur de } \\
\text { l'Augmentation } \\
\text { du rendement } \\
\text { (\$/ha) }\end{array}$ & RVC \\
\hline T0 & 0 & 0 & 0 & 2,1 & - & - & - \\
T1 & 315 & 0 & 315 & 3,8 & 1,7 & 425 & 1,3 \\
T2 & 630 & 0 & 630 & 5,9 & 3,8 & 950 & 1,5 \\
T3 & 0 & 168 & 168 & 3,9 & 1,8 & 450 & 2,67 \\
T4 & 0 & 336 & 336 & 4,5 & 2,4 & 600 & 1,78 \\
T5 & 0 & 84 & 84 & 4,7 & 2,6 & 650 & 7,73 \\
T6 & 630 & 168 & 798 & 6,8 & 4,7 & 1175 & 1,47 \\
T7 & 630 & 336 & 966 & 7,7 & 5,6 & 1400 & 1,44 \\
T8 & 630 & 84 & 714 & 5,8 & 3,7 & 925 & 1,29 \\
T9 & 315 & 168 & 483 & 5,6 & 3,5 & 875 & 1,81 \\
T10 & 315 & 336 & 651 & 7,3 & 6,2 & 1550 & 2,38 \\
T11 & 315 & 84 & 399 & 5,3 & 3,2 & 800 & 2 \\
\hline
\end{tabular}

Légende : T0 : sans engrais minéral ni DHR ; T1 (sans DHR, $150 \mathrm{~kg} \mathrm{NPK+100} \mathrm{kg} \mathrm{urée)} \mathrm{;} \mathrm{T2} \mathrm{(sans} \mathrm{DHR,} 300 \mathrm{~kg} \mathrm{NPK+200} \mathrm{kg}$ urée) ; $\mathbf{T 3}$ (3,5 tonnes de DHR, sans engrais minéral) ; T4 (7 tha-1 de DHR, sans engrais minéral) ; T5 (1,75 tha-1 de DHR, sans engrais minéral) ; $T 6$ (3,5 tha-1 de DHR, $300 \mathrm{~kg} \mathrm{NPK}$ et $200 \mathrm{~kg}$ urée); $T 7$ (7 tha-1 de DHR, $300 \mathrm{~kg} \mathrm{NPK}$ et $200 \mathrm{~kg}$ urée) ; T8 (1,75 tha-1 de DHR, $300 \mathrm{~kg} \mathrm{NPK}$ et $200 \mathrm{~kg}$ urée) ; $\boldsymbol{T 9}$ (3,5 tha-1 de DHR, $150 \mathrm{~kg} \mathrm{NPK}$ et $100 \mathrm{~kg}$ urée) ; $\boldsymbol{T 1 0}$ (7 tha-1 de DHR, $150 \mathrm{~kg}$ NPK et $100 \mathrm{~kg}$ urée); $T 11$ (1,75 t.ha-1 de DHR, $150 \mathrm{~kg}$ NPK et $100 \mathrm{~kg}$ urée)

\section{DISCUSSION}

La comparaison entre les 3 variétés testées montre que Babungo, en dépit de sa sensibilité à la verse suite à la taille des plantes, a été cependant très productif. Les résultats obtenus par Useni et al. (2012) ont montré également que Unilu et Katanga donnent des rendements moins élevés que celui obtenu avec Unilu. Ceci montre ainsi l'importance des variétés améliorées dans l'intensification de l'agriculture. En effet, Alley et Vanlauwe (2009) ont préconisé qu'une gestion intégrée de la fertilité des sols devra s'appuyer sur l'utilisation des germoplasmes améliorés, l'utilisation des engrais minéraux, la bonne gestion de la matière organique $\mathrm{du}$ sol et les adaptations. SENASEM (2008) indique qu'en station, le rendement moyen est de l'ordre de 6 à 8 t.ha $^{-1}$ en RD Congo; ce qui se rapproche de la moyenne des rendements obtenu avec la variété Babungo. Une des contraintes majeures pour la gestion durable de la fertilité des terres dans le milieu paysan est le manque d'information sur les nutriments limitants du sol. Les résultats de cette étude montrent l'intérêt de l'apport combiné des déchets humains recyclés et des engrais minéraux sur la production du maîs. L'apport des engrais minéraux sans ajout des déchets humains recyclés n'a pas permis d'accroitre significativement le rendement, dans le contexte de cette étude. Les fortes doses de fertilisants induisent une augmentation du coût de production, entrainant une réduction du profit. L'utilisation de la matière organique combinée aux engrais chimiques est primordiale pour améliorer l'efficacité des engrais car la matière organique permet d'accroitre la capacité de rétention de l'eau (Mulaji, 2011) et des éléments nutritifs (Useni et al., 2013), permet une disponibilisation progressive des éléments nutritifs aux plantes, accroitre le taux des micronutriments (Kaho et al., 2011 ; Mukengere, 2010). Or en examinant la loi de Liebig, la déficience en un élément nutritif diminue la capacité d'utilisation des autres nutriments (Vanlauwe et al., 2006 ; Mukengere, 2010). Malheureusement la disponibilité de la matière organique chez les paysans africains n'est pas garantie (Titonell et al., 2005) et l'abondance des déchets humains recyclés est une voie prometteuse (Useni et al., 2013). Les rendements étaient faibles sur les parcelles fertilisées aux engrais minéraux. Ceci se justifierait par la faible efficience des engrais minéraux dans les sols acides de la région (Mukalay et al., 2008 ; Kasongo, 2008). Cette faible efficience agronomique limite l'utilisation de l'engrais dans les ménages des agriculteurs mais elle pourrait être améliorée grâce à une gestion complémentaire de la structure du sol et des matières organiques. II a était démontré que seulement 10 à $15 \%$ de $\mathrm{P}$, de 10 à $20 \%$ 
de l'azote appliqué à travers les engrais sont assimilé par la plante (Mukengere, 2010). Les déchets organiques jouent un rôle important dans la gestion d'une meilleure fertilité du sol en améliorant sa structure (Mulaji, 2011). La combinaison d'engrais organique et minéraux crée les meilleurs conditions de production car la matière organique améliore les propriétés du sol alors que les engrais minéraux apportent aux plantes les éléments nutritifs qui leurs sont nécessaires et d'accroitre l'efficacité agronomique (Mukengere, 2010). Les résultats obtenus ont montré que l'utilisation des fertilisants organiques accroit le rendement du maïs comparativement au traitement de référence et pour une meilleure production de maïs, les traitements à dose de 7 tonnes de déchets humains recyclés associées à $150 \mathrm{~kg}$ de NPK et $100 \mathrm{~kg}$ d'urée est à souhaiter. L'analyse des rendements comparatifs obtenus avec différentes formes de fertilisation avec et sans fertilisants organiques fait ressortir les principaux éléments suivants : la fumure organique permet d'augmenter les rendements par rapport à des sols non fertilisés, même lorsqu'il est appliqué à de faibles doses; le type des composts et les doses appliquées influencent directement les rendements; les applications de fertilisants organiques produisent une diminution de la fréquence d'utilisation des engrais minéraux (Jama et al., 2000 ; Amuyojegbe et al., 2007 ; Muna-Mucheru et al., 2007 ; Mulaji, 2011 ; Useni et al., 2012 ; Kasongo et al., 2013; Useni et al., 2013). Dans une étude de Yamika et Ikawati (2012) évaluant les effets combinés de 3 doses de fertilisants inorganiques

\section{CONCLUSION}

Les résultats obtenus ont montré que l'utilisation des fertilisants organiques accroit le rendement du maïs comparativement au traitement de référence et pour une meilleure production de maïs, les traitements à dose de 7 tonnes de déchets humains recyclés associées à $150 \mathrm{~kg}$ de NPK et $100 \mathrm{~kg}$ d'urée est à souhaiter. Le rendement moyen en grains de maïs augmente avec l'ajout des engrais minéraux aux fèces humaines. Par contre, la rentabilité économique diminue avec les doses croissantes d'engrais

\section{REFERENCES BIBLIOGRAPHIQUES}

Abedi T., Alemzadeh A., Kazemeini S.A., 2010. Effect of organic and inorganic fertilizers on grain yield and protein banding pattern of wheat. Australian journal of crop science, 4(6):384389
(75 kg ha-1, $\left.150 \mathrm{~kg} \mathrm{ha}^{-1}, 225 \mathrm{~kg} \mathrm{ha}^{-1}\right)$ et 3 doses de fumure organique $\left(0 \mathrm{t}^{-h a^{-1}}, 0.5 \mathrm{tha}^{-1}\right.$ et $\left.1 \mathrm{t} \mathrm{ha} \mathrm{a}^{-1}\right)$ ont montré que la combinaison de $225 \mathrm{~kg}^{-h^{-1}}$ et 1 tonne a permis d'accroitre le rendement du soja de $46 \%$ et la teneur en carbone organique du sol de $26 \%$, par rapport à la fertilisation minérale seule. Des résultats similaires ont été obtenus sur le soja et le maïs au Nigeria par Amuyojegbe et al. (2007), sur le maïs au Nigeria par Adeniyan (1998) et Uwah et al. (2011), sur le blé en Iran par Abedi et al. (2010) et sur le maïs et le chou de chine à Meghalaya par Islam et Munda (2012). La dose de 7 tha $^{-1}$ de déchets humains recyclés combinée à la forte dose des engrais minéraux donne le rendement maximal de 7,7 t.ha $^{-1}$ tandis que la dose minimale de 1,75 t.ha $^{-1}$ sans combinaison des engrais minéraux est plus rentable $(R V C=7,73)$. En outre, la rentabilité de la fertilisation organique a été comparable à celle de la fertilisation minérale et organominérale, sauf pour la faible dose des déchets humains recyclés. Lorsque les fortes doses des fertilisants sont utilisées, l'augmentation de rendement qu'elles entrainent ne fait que compenser les dépenses engagées pour leur achat (Useni et al., 2012). Les études similaires conduites au Kenya par Muna-Mucheru et al. (2007) et Jama et al. (2000), au Cameroun par Kaho et al. (2011) et à Lubumbashi par Useni et al. (2012) ont également montré que les traitements aux fertilisants organiques seuls procuraient les marges bénéficiaires les plus élevées que les traitements avec engrais minéraux seuls ou combinés aux fertilisants organiques.

organiques et l'ajout des engrais minéraux : La dose de 7 t.ha $^{-1}$ de déchets humains recyclés combinée à la forte dose des engrais minéraux donne le rendement maximal de 7,7 tha $^{-1}$ tandis que la dose minimale de 1,75 tha-1 sans combinaison des engrais minéraux est plus rentable $(\mathrm{RVC}=7,73)$. En outre, la rentabilité de la fertilisation organique a été comparable à celle de la fertilisation minérale et organominérale, sauf pour la faible dose des déchets humains recyclés.

Adeniyan O.N., 1998. The effect of combined use of poultry manure and NPK fertilizer on soil fertility and maize yield. Master thesis, Federal University of technology, Akure, Ondo state, Nigeria. 
Alley M.M., Vanlauwe B., 2009. The role of fertilizer in integrated plant nutrient management, IFA and TSBF-CIAT, Paris, France, 59p

Amujoyegbe B.J., Opabode J.T., Olayinka A., 2007. Effect of organic and inorganic fertilizer on yield and chlorophyll content of maize (Zea mays L.) and sorghum (Sorghum bicolour (L.) Moench). African Journal of Biotechnology 6 (16) : 1869-1873

Anzala F., 2006. Contrôle de la vitesse de germination chez le Maïs (Zea mays) : Étude de la voie de biosynthèse des acides aminés issus de l'aspartate et recherche de QTLs. Thèse de doctorat, Ecole doctorale d'Angers, 186p.

FAO, 2000. Fertilizers and their use - A pocket guide for extension officers. Fourth edition. FAO, Rome

Galla T.J., Camara M., Yao Kouame A., Keli Z.J., 2011. Rentabilité des engrais minéraux en riziculture de plateau : cas de la zone de Gagnoa dans le centre ouest de la Cote d'Ivoire. Journal of applied bioscience 46: 3153-3162

GROUPE HUIT, 2009. Elaboration du plan urbain de référence de Lubumbashi. Rapport final Groupe Huit, BEAU, Ministère des ITR, RD Congo, $62 p$

Islam M., Munda G.C., 2012. Effect of organic and inorganic fertilizer on growth, productivity, nutrient uptake and economics of maize (Zea mays L.) and toria (Brassica campestris L.). Agricultural Science Research Journals Vol. 2(8): 470-479

Jama B., Palm C.A., Buresh R.J., Niang A.I., Gachengo C., Nziguheba G., 2000. Tithonia as a green manure for soil fertility improvement in Western Kenya: a review. Agroforestry Systems, 49: 201-221.

Kaho F., Yemefack M., Feujio-Tegwefouet P., Tchanthaouang J.C., 2011. Effet combiné de feuilles de Tithonia diversifolia et des engrais inorganiques sur le rendement du maïs et les propriétés d'un sol ferralitique au centre du Cameroun. Tropicultura, 29 (1): 39-45

Kasongo L.E., 2008. Système d'évaluation des terres à multiples échelles pour la détermination de l'impact de la gestion agricole sur la sécurité alimentaire au Katanga R.D.Congo. Thèse de doctorat, Université de Gand Belgique 336p.

Kasongo L.M.E., Mwamba M.T., Tshipoya M.P., Mukalay M.J., Useni S.Y., Mazinga K.M., Nyembo K.L., 2013. Réponse de la culture de soja (Glycine max L. (Merril) à l'apport des biomasses vertes de Tithonia diversifolia (Hemsley) A. Gray comme fumure organique sur un Ferralsol à Lubumbashi, R.D. Congo. Journal of Applied Biosciences 63: 4727 4735

Kiba D.I., Compaoré E., Nacro H.B., Lompo F., Sedogo M.P., 2008. Valorisation agronomique de déchets d'abattoir et de décharges de la ville de Ouagadougou (Burkina Faso). Rapport de I'INERA et l'IDR et l'UPB, Burkina Faso.

Mukalay M.J., Shutcha M.N., Tshomba K.J., Mulowayi K., Kamb C.F., Ngongo L.M., 2008. Causes d'une forte hétérogénéité des plants dans un champ de maïs dans les conditions pédoclimatique de Lubumbashi. Presses universitaires de Lubumbashi, Annales Faculté des Sciences Agronomiques, vol 1, $n^{\circ} 2: 4-11$

Mukengere B.E., 2010. Evaluation de l'efficacité d'usage des engrais dans les sols dégradés du Sud-Kivu sur la culture du maïs et du haricot. Cas du groupement de Burhale. Mémoire de fin d'études, Faculté des Sciences Agronomiques et environnement, Université Evangélique en Afrique, 59p

Mulaji K.C., 2011. Utilisation des composts de biodéchets ménagers pour l'amélioration de la fertilité des sols acides de la province de Kinshasa (République Démocratique du Congo). Thèse de doctorat, Gembloux Agro bio tech, 220p.

Muna-Mucheru M., Mugendi D., Kung'u J., Mugwe J., Bationo A., 2007. Effects of organic manure and mineral fertilizer inputs on maize yield and soil chemical properties in a maize cropping system in Meru South District, Kenya. Agroforestry Systems, 69:189-197.

N'Dienor M., 2006. Fertilité et gestion de la fertilisation dans les systèmes maraîchers périurbains des pays en développement : intérêts et limites de la valorisation agricole des déchets urbains dans ces systèmes, cas de l'agglomération d'Antananarivo (Madagascar). Thèse de doctorat, Université d'Antananarivo, Ecole Supérieure des Sciences Agronomiques (ESSA), 242p

Nyembo K.L., 2010. Augmentation du rendement $d u$ maïs par l'exploitation de l'effet hétérosis des hybrides produits au Katanga, République Démocratique du Congo. Thèse de doctorat, 
Faculté des sciences agronomiques, Université de Lubumbashi, $157 \mathrm{p}$.

SENASEM, 2008. Catalogue variétal des cultures vivrières : Céréales (maïs, riz), Légumineuses (haricot, soja, niébé), Plantes à tubercules (manioc, patate douce, pomme de terre), Bananier. Appui du projet CTB/MINAGRI, Kinshasa, 153p.

Tittonell P., Vanlauwe B., Leffelaar P.A., Shepherd K.D., Giller K.E., 2005. Exploring diversity in soil fertility management of smallholder farms in western Kenya. Within farm variability in resource allocation, nutrient flows and soil fertility status. Agriculture Ecosystem Environnement 110:166-184

Useni S.Y, Baboy L.L., Nyembo K.L., Mpundu M.M., 2012. Effets des apports combinés de biodéchets et de fertilisants inorganiques sur le rendement de trois variétés de Zea mays $L$. cultivées dans la région de Lubumbashi. Journal of Applied Biosciences 54: 39353943

Useni S.Y., Chukiyabo K.M., Tshomba K.J., Muyambo M.E., Kapalanga K.P., Ntumba N.F., Kasangij A-K.P., Kyungu K.A., Baboy L.L., Nyembo K.L., Mpundu M.M., 2013. Utilisation des déchets humains recyclés pour l'augmentation de la production du maïs (Zea mays L.) sur un ferralsol du sud-est de la RD Congo. Journal of Applied Biosciences 66:5070 - 5081

Uwah D.F., Eneji A.E., Eshiet U.J., 2011. Organic and mineral fertilizers effects on the performance of sweet maize (Zea mays L. saccharata strut.) in south eastern rainforest zone of Nigeria. International Journal of Agriculture Sciences, vol. $3\left(n^{\circ} 1\right): 54-61$

Vanlauwe B., Giller K.E., 2006. Popular myths around soil fertility management in sub-Saharan Africa. Agriculture Ecosystem Environnement 116:34-46p

Yamika W.S.D., Ikawati K.R., 2012. Combination inorganic and organic fertilizer increased yield production of soybean In Rain-Field Malang, Indonesia. American-Eurasian Journal of Sustainable Agriculture, 6(1): 14-17 